\title{
Anaemia and Iron Homeostasis in a Cohort of HIV-Infected Patients: A Cross-Sectional Study in Ghana
}

\author{
Christian Obirikorang, ${ }^{1}$ Razak Gyesi Issahaku, ${ }^{1,2}$ \\ Derick Nii Mensah Osakunor, ${ }^{1}$ and James Osei-Yeboah ${ }^{3}$ \\ ${ }^{1}$ Department of Molecular Medicine, School of Medical Sciences, College of Health Sciences, \\ Kwame Nkrumah University of Science and Technology, Kumasi, Ghana \\ ${ }^{2}$ Medical Laboratory Department, Tamale Teaching Hospital, Tamale, Ghana \\ ${ }^{3}$ Department of Medical Laboratory Sciences, University of Health and Allied Sciences, Ho, Ghana \\ Correspondence should be addressed to Derick Nii Mensah Osakunor; osakunor@gmail.com
}

Received 19 November 2015; Revised 29 January 2016; Accepted 1 March 2016

Academic Editor: Robert R. Redfield

Copyright (C) 2016 Christian Obirikorang et al. This is an open access article distributed under the Creative Commons Attribution License, which permits unrestricted use, distribution, and reproduction in any medium, provided the original work is properly cited.

\begin{abstract}
Aim. We determined the prevalence of anaemia and evaluated markers of iron homeostasis in a cohort of HIV patients. Methods. A comparative cross-sectional study on 319 participants was carried out at the Tamale Teaching Hospital from July 2013 to December 2013, 219 patients on HAART (designated On-HAART) and 100 HAART-naive patients. Data gathered include sociodemography, clinical history, and selected laboratory assays. Results. Prevalence of anaemia was $23.8 \%$. On-HAART participants had higher CD4/CD3 lymphocyte counts, Hb, HCT/PCV, MCV, MCH, iron, ferritin, and TSAT $(P<0.05)$. Hb, iron, ferritin, and TSAT decreased from grade 1 to grade 3 anaemia and CD4/CD3 lymphocyte count was lowest in grade 3 anaemia $(P<0.05)$. Iron $(P=0.0072)$ decreased with disease severity whilst transferrin $(P=0.0143)$ and TIBC $(P=0.0143)$ increased with disease severity. Seventy-six (23.8\%) participants fulfilled the criteria for anaemia, $86(26.9 \%)$ for iron deficiency, 41 (12.8\%) for iron deficiency anaemia, and 17 (5.3\%) for iron overload. The frequency of anaemia was higher amongst participants not on HAART (OR 2.6 for grade 1 anaemia; OR 3.0 for grade 3 anaemia). Conclusion. In this study population, HIV-associated anaemia is common and is related to HAART status and disease progression. HIV itself is the most important cause of anaemia and treatment of HIV should be a priority compared to iron supplementation.
\end{abstract}

\section{Introduction}

Iron deficiency is the leading cause of anaemia in the developing world [1]; the World Health Organization (WHO) and World Bank have ranked iron deficiency anaemia as the third leading cause of Disability-Adjusted Life Years (DALYs) lost for females (15-44 years) and as part of top 10 disease burdens for men [2]. The condition has also been classified as a severe public health problem in children and for pregnant women in more than 67 countries [3].

At the end of 2012, an estimated 35.3 million people worldwide were living with HIV and Sub-Saharan Africa was the region most heavily affected $[4,5]$. Anaemia is a common feature of HIV infection (20-80\%) [6] and occurs in about
$35 \%$ of patients who initiate Highly Active Antiretroviral Therapy (HAART) [7].

Anaemia at the time of HAART initiation has been associated with HIV disease progression and mortality [6, 812]. O'Brien et al. [12] in a recent study found that moderate and severe anaemia were associated with an increased mortality among Tanzanian women with HIV. This was true after controlling for potential confounders including CD4 cell count, clinical stage, and body mass index. As HIV disease severity progresses, the likelihood of developing anaemia increases [6], thus impacting negatively the quality of life of HIV patients. A study in 2009 by Obirikorang and Yeboah showed that the association between anaemia and mortality is causal and anaemia acts as a surrogate marker of 
the underlying disease, HIV [13]. In the developing world, several other etiologic factors such as micronutrient deficiencies and blood loss from intestinal opportunistic disease may also be involved in the development of HIV-associated anaemia [14].

So far, data on the levels of various markers of iron status in HIV are limited and in other instances are contradicting. High plasma ferritin concentrations have been found among HIV-infected patients [15-17] while other studies have reported low ferritin concentrations [18, 19]. Treatment regimens in the developed world may include erythropoietin; the high cost of this however restricts its use in resource-limited settings [20]. Studies from Europe and North America have shown that HAART can be an effective treatment for anaemia of HIV infection [21-23]. Whether the same holds true in Ghana and Africa, where comorbidities such as micronutrient deficiencies, malaria, TB, and parasitic infections are common, remains to be shown.

In this study, we determined the prevalence of anaemia in a cohort of HIV patients in a developing country and measured markers of iron homeostasis in relation to anaemia, disease progression, and HAART status.

\section{Methodology}

2.1. Study Design/Site. This comparative cross-sectional study was carried out at the Sexually Transmitted Infections (STI) clinic of the Tamale Teaching Hospital from July 2013 to December 2013. The hospital is located in the Tamale Metropolis of the Northern Region of Ghana. The hospital was established to serve as a medical referral centre for the Northern, Upper East, Upper West, and parts of the Brong Ahafo Regions of Ghana and also for neighbouring countries: La Cote d'Ivoire, Burkina Faso, and Togo.

2.2. Ethical Consent. The study was approved by the Committee on Human Research, Publication and Ethics (CHRPE) of the School of Medical Sciences (SMS), Kwame Nkrumah University of Science and Technology (KNUST), and the Department of Research and Monitoring, Tamale Teaching Hospital. Participation was voluntary and written informed consent was obtained from each participant.

2.3. Study Population. At the time of first presentation at the hospital, all patients (both HAART-naive and On-HAART) have a "baseline visit" for a structured interview and laboratory examinations, after which they come for scheduled visits, as requested by the attending physician. Three hundred and nineteen participants were recruited for the study, which consisted of 219 patients on HAART (designated On-HAART) and 100 HAART-naive patients (designated HAART-naive). Patients were included in the study if they were diagnosed and confirmed HIV-positive, aged $\geq 18$ years, followed up by the Tamale Teaching Hospital STI clinic, on HAART for at least three (3) months, and with good adherence to therapy. Good adherence was defined as missing $<2$ doses of 30 doses or $<3$ doses of 60 doses [24]. Patients who were pregnant and those with inflammation, defined as a CRP $>8.2 \mathrm{mg} / \mathrm{L}$ [25], were excluded.
2.4. Data Collection and Laboratory Methods. An interviewbased questionnaire was used to gather data on sociodemography. Clinical history was obtained from patient records.

Five $\mathrm{mL}$ of early morning venous blood was drawn from each participant; $2 \mathrm{~mL}$ was dispensed into a vacutainer tube containing ethylenediaminetetraacetic acid (EDTA) and $3 \mathrm{~mL}$ was dispensed into a serum separator tube (SST), allowed to clot, and then centrifuged at $3000 \mathrm{~g}$ for 5 minutes. Aliquots of the serum were stored at $-80^{\circ} \mathrm{C}$ until assays were performed. Laboratory assays included CD4/CD3 lymphocyte counts by flow cytometry (BD FACSCOUNT, Becton Dickenson and Company, California, USA) and haemoglobin and red/white cell indices (Mindray BC 3000 Plus, Mindray Company, Shenzhen, China). Serum iron, ferritin, transferrin, and transferrin saturation (TSAT) were measured using the Flexor XL analyser from vital scientific. Serum CRP was also performed with a semiquantitative immunechromatographic method, to guide in excluding a rise in serum ferritin due to acute inflammation [26].

2.5. Data Analysis and Statistics. Disease progression as indicated by CD 4 count was Stage 1 ( $\geq 500$ cells $/ \mathrm{mm}^{3}$ ), Stage 2 $\left(200-499\right.$ cells $\left./ \mathrm{mm}^{3}\right)$, and Stage $3\left(<200\right.$ cells $\left./ \mathrm{mm}^{3}\right)$ as per recommendations of the Center for Disease Control (CDC) [27-29]. The World Health Organization/Association of Clinical Trial Group (WHO/ACTG) criteria were used to define mild (Hb 10.5-12.99 g/dL for men; 10.5-11.99 g/dL for women), moderate ( $\mathrm{Hb} 8.0-10.49 \mathrm{~g} / \mathrm{dL})$, and severe $(\mathrm{Hb}<$ $8.0 \mathrm{~g} / \mathrm{dL})$ anaemia $[30,31]$. Using the Mean Cell Volume (MCV), reference limits of $80-96 \mathrm{fL}$ and Mean Cell Haemoglobin $(\mathrm{MCH})$ reference limits of $27-32 \mathrm{pg}$ as pointers, low MCV (<80 fL) was indicative of microcytosis, high $\mathrm{MCV}$ (>96 fL) was indicative of macrocytosis, and low $\mathrm{MCH}$ $(<27 \mathrm{pg})$ indicates hypochromia [32]. Iron deficiency (ID) was defined as serum iron $<8.6 \mu \mathrm{mol} / \mathrm{L}$, ferritin $<15 \mu \mathrm{g} / \mathrm{L}$, and TSAT $<16 \%$. Iron deficiency anaemia (IDA) was defined as $\mathrm{Hb} \leq 10.5 \mathrm{~g} / \mathrm{dL}$, serum iron $\leq 8.6 \mu \mathrm{mol} / \mathrm{L}$, ferritin $<15 \mu \mathrm{g} / \mathrm{L}$, and TSAT $<16 \%$. Iron overload was defined as serum iron $>$ $30 \mu \mathrm{mol} / \mathrm{L}$, ferritin $>300 \mu \mathrm{g} / \mathrm{L}$, and TSAT $>55 \%$.

Data are presented as mean \pm standard deviation (SD) or $n$ (\%). Unpaired $t$-test was used to compare the means of all continuous variables. Categorical data were analysed using Fisher's exact test or Chi-square where applicable. Multiple variables were analysed using the one-way ANOVA. For all statistical comparisons, a $P$ value of $<0.05$ was considered significant. Data were analysed using GraphPad Prism version 6.0 for windows (GraphPad software, San Diego, California, USA).

\section{Results}

Of the total, there were significantly more females than males on HAART $(P=0.0028)$ (Table 1$)$.

On-HAART participants had significantly higher CD4/ CD3 lymphocyte counts, $\mathrm{Hb}$, haematocrit, $\mathrm{MCV}, \mathrm{MCH}$, RDW-SD, serum iron, ferritin, and transferrin saturation than when compared to their corresponding HAART-naive group $(P<0.05)$. WBC count (within normal limits of reference), serum transferrin, and TIBC were however higher amongst the HAART-naive individuals $(P<0.05)$ (Table 2$)$. 
TABLE 1: Sociodemographic characteristic of the study population.

\begin{tabular}{|c|c|c|c|c|}
\hline Parameter & Total $(n=319)$ & On-HAART $(n=219)$ & HAART-naive $(n=100)$ & $P$ value \\
\hline \multicolumn{5}{|l|}{ Gender $n(\%)$} \\
\hline Female & $217(68.0)$ & $161(73.5)$ & $56(56.0)$ & \multirow[t]{2}{*}{0.0028} \\
\hline Male & $102(32.0)$ & $58(26.5)$ & $44(44.0)$ & \\
\hline \multicolumn{5}{|l|}{ Age (yrs) } \\
\hline Mean \pm SD & $38.89 \pm 9.91$ & $39.93 \pm 9.72$ & $37.91 \pm 9.53$ & 0.0842 \\
\hline $18-24 n(\%)$ & $25(7.8)$ & $11(5.0)$ & $14(14.0)$ & \multirow[t]{5}{*}{0.0076} \\
\hline $25-34 n(\%)$ & $96(30.1)$ & $62(28.3)$ & $34(34.0)$ & \\
\hline $35-44 n(\%)$ & $108(33.9)$ & $83(37.9)$ & $25(25.0)$ & \\
\hline $45-54 n(\%)$ & 55 (17.2) & $42(19.1)$ & $13(13.0)$ & \\
\hline$>54 n(\%)$ & $35(11.0)$ & $21(9.6)$ & $14(14.0)$ & \\
\hline \multicolumn{5}{|l|}{ Marital status $n(\%)$} \\
\hline Married & $198(62.1)$ & $142(64.8)$ & $56(56.0)$ & \multirow[t]{2}{*}{0.1375} \\
\hline Single & $121(37.9)$ & $77(35.2)$ & $44(44.0)$ & \\
\hline \multicolumn{5}{|c|}{ Educational level $n(\%)$} \\
\hline No education & $106(33.2)$ & $76(34.7)$ & $30(30.0)$ & \\
\hline Basic education & $92(28.8)$ & $69(31.5)$ & $23(23.0)$ & \\
\hline SSS & $65(20.4)$ & $39(17.8)$ & $26(26.0)$ & \multirow[t]{2}{*}{0.1432} \\
\hline Tertiary & $56(17.6)$ & $35(16.0)$ & $21(21.0)$ & \\
\hline \multicolumn{5}{|c|}{ Employment status $n(\%)$} \\
\hline Formal & $73(22.9)$ & $47(21.5)$ & $26(26.0)$ & \multirow[t]{3}{*}{0.0591} \\
\hline Informal & $175(54.9)$ & $129(58.9)$ & $46(46.0)$ & \\
\hline Unemployed & $71(22.3)$ & 43 (19.6) & $28(28.0)$ & \\
\hline
\end{tabular}

Data are presented as mean $\pm \mathrm{SD}$ or $n(\%) . P$ values represent comparisons between On-HAART and HAART-naïve participants. $P$ values $<0.05$ were considered significant. HAART = Highly Active Antiretroviral Therapy.

TABLE 2: Immunological, haematological, and biochemical profiles of the study population stratified by HAART status.

\begin{tabular}{|c|c|c|c|c|}
\hline Parameter & Total $(n=319)$ & On-HAART $(n=219)$ & HAART-naive $(n=100)$ & $P$ value \\
\hline \multicolumn{5}{|c|}{ Immunological profile $($ mean \pm SD) } \\
\hline CD4 (cells $\left./ \mathrm{mm}^{3}\right)$ & $426.40 \pm 191.40$ & $475.40 \pm 229.50$ & $207.10 \pm 102.20$ & $<0.0001$ \\
\hline CD3 (cells/mm³ & $1329.00 \pm 513.70$ & $1394.00 \pm 403.9$ & $1036.00 \pm 377.2$ & 0.0007 \\
\hline $\mathrm{CD} 4 / \mathrm{CD} 3$ & $0.31 \pm 0.12$ & $0.34 \pm 0.12$ & $0.20 \pm 0.56$ & $<0.0001$ \\
\hline $\mathrm{WBC}\left(10^{3} / \mu \mathrm{L}\right)$ & $5.23 \pm 1.86$ & $5.09 \pm 1.81$ & $5.88 \pm 1.70$ & 0.0151 \\
\hline \multicolumn{5}{|c|}{ Haematological profile (mean \pm SD) } \\
\hline $\mathrm{Hb}(\mathrm{g} / \mathrm{dL})$ & $11.65 \pm 1.70$ & $11.89 \pm 1.56$ & $10.59 \pm 1.88$ & $<0.0001$ \\
\hline $\operatorname{RBC}\left(10^{6} / \mu \mathrm{L}\right)$ & $3.86 \pm 0.58$ & $3.84 \pm 0.58$ & $3.93 \pm 0.62$ & 0.3576 \\
\hline $\operatorname{HCT}(\%)$ & $35.01 \pm 4.76$ & $35.67 \pm 4.41$ & $32.04 \pm 5.18$ & $<0.0001$ \\
\hline $\mathrm{MCV}(\mathrm{fL})$ & $91.82 \pm 11.73$ & $97.99 \pm 10.96$ & $82.09 \pm 10.08$ & $<0.0001$ \\
\hline $\mathrm{MCH}(\mathrm{pg})$ & $31.79 \pm 5.14$ & $32.67 \pm 4.97$ & $27.87 \pm 3.98$ & $<0.0001$ \\
\hline $\mathrm{MCHC}(\mathrm{g} / \mathrm{dL})$ & $34.62 \pm 2.83$ & $34.75 \pm 2.78$ & $34.03 \pm 2.99$ & 0.1433 \\
\hline RDW-CV & $15.17 \pm 1.76$ & $14.96 \pm 1.57$ & $16.13 \pm 2.25$ & 0.0001 \\
\hline RDW-SD & $52.20 \pm 7.28$ & $52.82 \pm 7.41$ & $49.40 \pm 5.99$ & 0.0068 \\
\hline $\operatorname{PLT}\left(10^{3} / \mu \mathrm{L}\right)$ & $242.3 \pm 78.75$ & $240.50 \pm 71.32$ & $250.40 \pm 106.60$ & 0.4756 \\
\hline \multicolumn{5}{|c|}{ Biochemical profile (mean $\pm \mathrm{SD})$} \\
\hline Iron $(\mu \mathrm{mol} / \mathrm{L})$ & $13.63 \pm 11.73$ & $14.51 \pm 12.40$ & $9.70 \pm 3.94$ & 0.0187 \\
\hline Ferritin $(\mu \mathrm{g} / \mathrm{L})$ & $255.00 \pm 51.48$ & $265.20 \pm 89.96$ & $238.10 \pm 57.45$ & 0.0691 \\
\hline Transferrin (mg/dL) & $203.90 \pm 36.81$ & $199.60 \pm 30.28$ & $223.20 \pm 54.05$ & 0.0002 \\
\hline TIBC $(\mu \mathrm{g} / \mathrm{dL})$ & $259.00 \pm 46.75$ & $253.50 \pm 38.45$ & $283.50 \pm 68.64$ & 0.0002 \\
\hline$\%$ TSAT & $30.82 \pm 27.08$ & $33.00 \pm 18.57$ & $21.06 \pm 10.85$ & 0.0114 \\
\hline
\end{tabular}

Data are presented as mean \pm SD. $P$ values represent comparisons between On-HAART and HAART-naive participants. $P$ values $<0.05$ were considered significant. $\mathrm{WBC}=$ White Blood Cells, $\mathrm{Hb}=$ haemoglobin, $\mathrm{RBC}=$ Red Blood Cells, $\mathrm{HCT}=$ haematocrit, $\mathrm{MCV}=\mathrm{Mean}$ Cell Volume, $\mathrm{MCH}=\mathrm{Mean}$ Cell Haemoglobin, $\mathrm{MCHC}=$ Mean Cell Haemoglobin Concentration, RDW-CV $=$ Red Cell Distribution Width-Cumulative Variance, RDW-SD = Red Cell Distribution Width-Standard Deviation, PLT $=$ platelets, TIBC $=$ Total Iron Binding Capacity, TSAT $=$ transferrin saturation, and HAART $=$ Highly Active Antiretroviral Therapy. 
TABLE 3: Immunological, haematological, and biochemical profiles of the study population stratified by anaemia status.

\begin{tabular}{|c|c|c|c|}
\hline Parameter & Anaemia $(n=76)$ & No anaemia $(n=243)$ & $P$ value \\
\hline Age (yrs) & $36.87 \pm 11.25$ & $39.51 \pm 9.40$ & 0.0924 \\
\hline \multicolumn{4}{|c|}{ Immunological profile $($ mean \pm SD) } \\
\hline CD4 (cells $\left./ \mathrm{mm}^{3}\right)$ & $264.50 \pm 98.40$ & $476.80 \pm 164.30$ & $<0.0001$ \\
\hline CD3 (cells $\left./ \mathrm{mm}^{3}\right)$ & $1029.00 \pm 485.20$ & $1422.00 \pm 559.90$ & $<0.0001$ \\
\hline $\mathrm{CD} 4 / \mathrm{CD} 3$ & $0.23 \pm 0.04$ & $0.34 \pm 0.13$ & $<0.0001$ \\
\hline $\mathrm{WBC}\left(10^{3} / \mu \mathrm{L}\right)$ & $5.06 \pm 2.01$ & $5.29 \pm 1.18$ & 0.4585 \\
\hline \multicolumn{4}{|c|}{ Haematological profile (mean \pm SD) } \\
\hline $\mathrm{Hb}(\mathrm{g} / \mathrm{dL})$ & $9.32 \pm 1.17$ & $12.38 \pm 1.06$ & $<0.0001$ \\
\hline $\mathrm{RBC}\left(10^{6} / \mu \mathrm{L}\right)$ & $3.43 \pm 0.52$ & $3.99 \pm 0.54$ & 0.5029 \\
\hline $\operatorname{HCT}(\%)$ & $28.89 \pm 3.56$ & $36.91 \pm 3.24$ & $<0.0001$ \\
\hline MCV (fL) & $85.41 \pm 12.13$ & $93.81 \pm 10.89$ & $<0.0001$ \\
\hline $\mathrm{MCH}(\mathrm{pg})$ & $28.39 \pm 5.30$ & $32.85 \pm 4.62$ & $<0.0001$ \\
\hline $\mathrm{MCHC}(\mathrm{g} / \mathrm{dL})$ & $33.24 \pm 3.21$ & $35.05 \pm 2.56$ & 0.0017 \\
\hline RDW-CV & $16.61 \pm 2.16$ & $14.73 \pm 1.34$ & $<0.0001$ \\
\hline RDW-SD & $52.59 \pm 8.49$ & $52.07 \pm 6.88$ & 0.3865 \\
\hline $\operatorname{PLT}\left(10^{3} / \mu \mathrm{L}\right)$ & $251.50 \pm 91.12$ & $239.40 \pm 74.56$ & 0.3357 \\
\hline \multicolumn{4}{|c|}{ Biochemical profile (mean \pm SD) } \\
\hline Iron $(\mu \mathrm{mol} / \mathrm{L})$ & $8.93 \pm 3.99$ & $15.09 \pm 6.52$ & 0.0019 \\
\hline Ferritin $(\mu \mathrm{g} / \mathrm{L})$ & $228.70 \pm 56.48$ & $270.10 \pm 39.21$ & 0.0021 \\
\hline Transferrin $(\mathrm{mg} / \mathrm{dL})$ & $229.00 \pm 55.13$ & $196.10 \pm 24.23$ & $<0.0001$ \\
\hline TIBC $(\mu \mathrm{g} / \mathrm{dL})$ & $290.80 \pm 70.02$ & $249.10 \pm 30.77$ & $<0.0001$ \\
\hline$\%$ TSAT & $18.87 \pm 8.15$ & $35.54 \pm 15.83$ & 0.0002 \\
\hline
\end{tabular}

Data are presented as mean \pm SD. $P$ values $<0.05$ were considered significant. WBC $=$ White Blood Cells, $\mathrm{Hb}=$ haemoglobin, RBC $=$ Red Blood Cells, $\mathrm{HCT}=$ haematocrit, $\mathrm{MCV}=$ Mean Cell Volume, $\mathrm{MCH}=$ Mean Cell Haemoglobin, $\mathrm{MCHC}=$ Mean Cell Haemoglobin Concentration, RDW-CV $=$ Red Cell Distribution Width-Cumulative Variance, RDW-SD = Red Cell Distribution Width-Standard Deviation, PLT = platelets, TIBC = Total Iron Binding Capacity, $\mathrm{TSAT}=$ transferrin saturation, and HAART $=$ Highly Active Antiretroviral Therapy.

TABLE 4: Immunological, haematological, and biochemical profiles of the study population stratified by grade of anaemia.

\begin{tabular}{|c|c|c|c|c|}
\hline Parameter & Grade $1(n=41)$ & Grade $2(n=19)$ & Grade $3(n=16)$ & $P$ value \\
\hline Age (yrs) & $35.79 \pm 10.64$ & $42.36 \pm 14.66$ & $33.75 \pm 5.85$ & 0.0591 \\
\hline \multicolumn{5}{|c|}{ Immunological profile (mean \pm SD) } \\
\hline CD4 $\left(\right.$ cells $\left./ \mathrm{mm}^{3}\right)$ & $271.50 \pm 121.10$ & $327.60 \pm 135.40$ & $148.90 \pm 71.13^{* * * \dagger \dagger \dagger \dagger}$ & $<0.0001$ \\
\hline CD3 (cells $\left./ \mathrm{mm}^{3}\right)$ & $1027.00 \pm 485.7$ & $1050.00 \pm 465.21$ & $1011.00 \pm 407.20$ & 0.0008 \\
\hline $\mathrm{CD} 4 / \mathrm{CD} 3$ & $0.24 \pm 0.14$ & $0.27 \pm 0.07$ & $0.14 \pm 0.05^{* *+\dagger \dagger \dagger}$ & $<0.0001$ \\
\hline $\mathrm{WBC}\left(10^{3} / \mu \mathrm{L}\right)$ & $4.78 \pm 1.87$ & $5.58 \pm 2.22$ & $5.58 \pm 1.67$ & 0.4141 \\
\hline \multicolumn{5}{|c|}{ Haematological profile (mean \pm SD) } \\
\hline $\mathrm{Hb}(\mathrm{g} / \mathrm{dL})$ & $10.03 \pm 0.26$ & $8.92 \pm 0.48^{* * * *}$ & $6.95 \pm 0.54^{* * * * \dagger \dagger \dagger \dagger}$ & $<0.0001$ \\
\hline $\operatorname{RBC}\left(10^{6} / \mu \mathrm{L}\right)$ & $3.53 \pm 0.43$ & $3.50 \pm 0.61$ & $2.95 \pm 0.49^{* * *+\dagger}$ & $<0.0001$ \\
\hline $\operatorname{HCT}(\%)$ & $30.88 \pm 1.76$ & $27.68 \pm 2.32^{* * * *}$ & $22.39 \pm 1.13^{* * * *+\dagger \dagger \dagger}$ & $<0.0001$ \\
\hline MCV (fL) & $88.68 \pm 10.99$ & $81.26 \pm 13.29^{*}$ & $77.60 \pm 10.94^{* *}$ & $<0.0001$ \\
\hline $\mathrm{MCH}(\mathrm{pg})$ & $29.78 \pm 4.88$ & $27.34 \pm 5.59$ & $24.11 \pm 4.44^{* * *}$ & $<0.0001$ \\
\hline $\mathrm{MCHC}(\mathrm{g} / \mathrm{dL})$ & $33.61 \pm 2.99$ & $33.72 \pm 3.89$ & $31.03 \pm 2.41^{* * \dagger}$ & $<0.0001$ \\
\hline RDW-CV & $15.90 \pm 1.67$ & $17.01 \pm 2.07^{*}$ & $18.95 \pm 2.55^{* * * * \dagger}$ & $<0.0001$ \\
\hline RDW-SD & $52.69 \pm 8.64$ & $51.21 \pm 7.38$ & $54.11 \pm 10.01$ & 0.8142 \\
\hline $\operatorname{PLT}\left(10^{3} / \mu \mathrm{L}\right)$ & $237.90 \pm 79.93$ & $275.40 \pm 105.30$ & $274.90 \pm 99.49$ & 0.3053 \\
\hline \multicolumn{5}{|c|}{ Biochemical profile (mean $\pm \mathrm{SD})$} \\
\hline Iron $(\mu \mathrm{mol} / \mathrm{L})$ & $10.69 \pm 4.81$ & $6.46 \pm 2.41^{* * *}$ & $5.06 \pm 2.75^{* * * *}$ & 0.0041 \\
\hline Ferritin $(\mu \mathrm{g} / \mathrm{L})$ & $216.1 \pm 38.65$ & $196.80 \pm 75.50$ & $181.00 \pm 83.31^{*}$ & 0.2346 \\
\hline Transferrin $(\mathrm{mg} / \mathrm{dL})$ & $206.70 \pm 34.25$ & $234.30 \pm 53.28^{*}$ & $313.80 \pm 46.19^{* * * * \dagger \dagger \dagger \dagger}$ & 0.0654 \\
\hline $\operatorname{TIBC}(\mu \mathrm{g} / \mathrm{dL})$ & $262.50 \pm 43.49$ & $297.50 \pm 66.66^{*}$ & $398.50 \pm 58.66^{* * * * \dagger \dagger \dagger \dagger}$ & 0.0654 \\
\hline$\%$ TSAT & $23.38 \pm 9.12$ & $13.24 \pm 4.12^{* * * *}$ & $8.00 \pm 4.78^{* * * * \dagger \dagger}$ & 0.0207 \\
\hline
\end{tabular}

Data are presented as mean \pm SD. $P$ values were obtained using one-way ANOVA of all variables. $*$ represents comparisons with grade $1 . \dagger$ represents comparisons with grade $2 . * / \dagger=<0.05 . * * / \dagger \dagger=<0.01 . * * *=<0.001 . * * * * / \dagger \dagger \dagger \dagger=<0.0001 . P$ values $<0.05$ were considered significant. WBC $=$ White Blood Cells, $\mathrm{Hb}=$ haemoglobin, RBC $=$ Red Blood Cells, HCT $=$ haematocrit, MCV = Mean Cell Volume, MCH $=$ Mean Cell Haemoglobin, MCHC $=$ Mean Cell Haemoglobin Concentration, RDW-CV = Red Cell Distribution Width-Cumulative Variance, RDW-SD = Red Cell Distribution Width-Standard Deviation, PLT $=$ platelets, $\mathrm{TIBC}=$ Total Iron Binding Capacity, TSAT $=$ transferrin saturation, and HAART $=$ Highly Active Antiretroviral Therapy. 
TABLE 5: Immunological, haematological, and biochemical profiles of the study population stratified by disease stage.

\begin{tabular}{|c|c|c|c|c|}
\hline \multirow{2}{*}{ Parameter } & \multicolumn{4}{|c|}{$\mathrm{CD}^{+}$lymphocyte count, cell $/ \mathrm{mm}^{3}$} \\
\hline & Stage $1(\geq 500)$ & Stage $2(200-499)$ & Stage $3(<200)$ & $P$ value \\
\hline Age (yrs) & $38.04 \pm 9.49$ & $40.00 \pm 9.46$ & $38.24 \pm 11.13$ & 0.3871 \\
\hline \multicolumn{5}{|l|}{ Immunological profile } \\
\hline CD3 $\left(\right.$ cell $\left./ \mathrm{mm}^{3}\right)$ & $1775.00 \pm 550.40$ & $1273.00 \pm 484.20^{*}$ & $810.70 \pm 310.70^{* \dagger}$ & $<0.0001$ \\
\hline $\mathrm{CD} 4 / \mathrm{CD} 3$ & $0.43 \pm 0.09$ & $0.32 \pm 0.12^{*}$ & $0.14 \pm 0.06^{* \dagger}$ & $<0.0001$ \\
\hline $\mathrm{WBC}\left(10^{3} / \mu \mathrm{L}\right)$ & $5.56 \pm 1.65$ & $5.12 \pm 1.89$ & $4.97 \pm 2.04$ & 0.1597 \\
\hline \multicolumn{5}{|l|}{ Haematological profile } \\
\hline $\mathrm{Hb}(\mathrm{g} / \mathrm{dL})$ & $12.28 \pm 1.37$ & $11.92 \pm 1.38$ & $10.35 \pm 1.90^{* \dagger}$ & $<0.0001$ \\
\hline $\operatorname{RBC}\left(10^{6} / \mu \mathrm{L}\right)$ & $4.01 \pm 0.55$ & $3.85 \pm 0.53$ & $3.64 \pm 0.65^{*}$ & 0.0015 \\
\hline $\operatorname{HCT}(\%)$ & $36.67 \pm 3.74$ & $35.82 \pm 4.08$ & $31.42 \pm 5.21^{* \dagger}$ & $<0.0001$ \\
\hline MCV (fL) & $92.49 \pm 11.20$ & $94.06 \pm 11.67$ & $87.27 \pm 11.48^{* \dagger}$ & 0.0025 \\
\hline $\mathrm{MCH}(\mathrm{pg})$ & $32.55 \pm 5.48$ & $32.41 \pm 4.42$ & $29.75 \pm 5.30^{* \dagger}$ & 0.0027 \\
\hline $\mathrm{MCHC}(\mathrm{g} / \mathrm{dL})$ & $35.11 \pm 3.64$ & $34.54 \pm 2.63$ & $34.08 \pm 3.29$ & 0.1120 \\
\hline RDW-CV & $14.64 \pm 1.43$ & $15.01 \pm 1.55$ & $16.16 \pm 2.11^{* \dagger}$ & $<0.0001$ \\
\hline RDW-SD & $50.79 \pm 6.42$ & $52.74 \pm 7.64$ & $53.23 \pm 7.61$ & 0.1100 \\
\hline $\operatorname{PLT}\left(10^{3} / \mu \mathrm{L}\right)$ & $250.30 \pm 68.68$ & $238.80 \pm 75.83$ & $237.10 \pm 95.21$ & 0.5524 \\
\hline \multicolumn{5}{|l|}{ Biochemical profile } \\
\hline Iron $(\mu \mathrm{mol} / \mathrm{L})$ & $14.73 \pm 6.08$ & $15.32 \pm 12.08$ & $9.38 \pm 7.64^{* \dagger}$ & 0.0072 \\
\hline Ferritin $(\mu \mathrm{g} / \mathrm{L})$ & $260.80 \pm 48.03$ & $262.80 \pm 42.05$ & $266.00 \pm 103.10$ & 0.9022 \\
\hline Transferrin (mg/dL) & $197.00 \pm 25.38$ & $202.50 \pm 24.69$ & $215.70 \pm 58.18^{*}$ & 0.0143 \\
\hline $\operatorname{TIBC}(\mu \mathrm{g} / \mathrm{dL})$ & $250.20 \pm 32.20$ & $257.10 \pm 31.35$ & $273.90 \pm 73.88$ & 0.0143 \\
\hline$\%$ TSAT & $33.60 \pm 13.12$ & $34.18 \pm 15.26$ & $21.59 \pm 9.77^{* \dagger}$ & 0.0133 \\
\hline
\end{tabular}

Data are presented as mean \pm SD. $P$ values $<0.05$ were considered significant. $P$ values represent one-way ANOVA of all three variables. $*$ represents comparisons with Stage 1. $\dagger$ represents comparisons with Stage $2 . * / \dagger=<0.05 . \mathrm{WBC}=$ White Blood Cells, Hb $=$ haemoglobin, RBC $=$ Red Blood Cells, $\mathrm{HCT}=$ haematocrit, $\mathrm{MCV}=$ Mean Cell Volume, $\mathrm{MCH}=$ Mean Cell Haemoglobin, $\mathrm{MCHC}=$ Mean Cell Haemoglobin Concentration, RDW-CV $=$ Red Cell Distribution Width-Cumulative Variance, RDW-SD = Red Cell Distribution Width-Standard Deviation, PLT = platelets, TIBC = Total Iron Binding Capacity, TSAT $=$ transferrin saturation, and HAART $=$ Highly Active Antiretroviral Therapy.

Seventy-six participants had anaemia, representing $23.8 \%$ of the study population. When grouped based on haemoglobin concentration, participants with anaemia had a significantly lower CD4/CD3 lymphocyte count $(P<0.0001$ each) with corresponding lower mean values of red cell indices (HCT, $P<0.0001 ; \mathrm{MCV}, P<0.0001 ; \mathrm{MCH}$, $P<0.0001 ; \mathrm{MCHC}, P=0.0017)$. Likewise, serum iron $(P=0.0019)$, ferritin $(P=0.0021)$, and TSAT $(P=0.0002)$ were significantly lower in anaemic patients than in those without anaemia. Serum transferrin $(P<0.0001)$ and TIBC $(P<0.0001)$ were however higher in anaemic patients than in nonanaemic patients (Table 3 ).

There was a decreasing trend in mean values of haemoglobin and corresponding red cell indices from grade 1 to grade 3 anaemia. A similar trend was observed with serum iron, ferritin, and TSAT, but the reverse in the case of serum transferrin and TIBC (increased from grade 1 through grade 3). CD4/CD3 lymphocyte counts were lowest in grade 3 anaemia. There were individual differences between various parameters when compared within and across groups (Table 4).

In Table 5, participants are grouped according to the severity of disease using CD4. Immunological markers (CD4, $P<0.0001$; CD3, $P<0.0001$; WBC, $P=0.1597)$ and serum iron $(P=0.0072)$ decreased with severity of disease. Serum ferritin $(P=0.9022)$, transferrin $(P=$ $0.0143)$, and TIBC $(P=0.0143)$ increased with severity of disease. There were individual differences between various parameters when compared within and across groups.

Characteristics of study participants, grouped according to iron status, are shown in Table 6. From the definitive criteria used, $76(23.8 \%)$ participants fulfilled the criteria for anaemia, 86 (26.9\%) for iron deficiency, 41 (12.8\%) for iron deficiency anaemia, and 17 (5.3\%) for iron overload. Significant linear trends were observed for HB, HCT, iron, and TSAT.

The frequency of anaemia was higher amongst participants not on HAART (OR 2.6 for grade 1 anaemia; OR 3.0 times for grade 3 anaemia $(P<0.05)$ ) (Table 7$)$.

\section{Discussion}

In this study, we determined the prevalence of anaemia in a cohort of HIV patients and measured markers of iron homeostasis in relation to anaemia, disease progression, and HAART. We showed that, in this population, HIVassociated anaemia is common and is associated with disease progression. The frequency of anaemia was higher amongst participants not on HAART. 
TABLE 6: Immunological, haematological, and biochemical profiles of the population under study stratified by iron status.

\begin{tabular}{|c|c|c|c|c|c|c|}
\hline Parameter & Anaemia $(n=76)$ & Iron deficiency $(n=86)$ & $\begin{array}{c}\text { Iron deficiency } \\
\text { anaemia }(n=41)\end{array}$ & $\begin{array}{l}\text { Iron overload } \\
\quad(n=17)\end{array}$ & $P$ value & $\begin{array}{l}P \text { value } \\
\text { (trend) }\end{array}$ \\
\hline Age & $36.87 \pm 11.25$ & $38.23 \pm 11.41$ & $36.22 \pm 10.37$ & $38.85 \pm 6.52$ & 0.8002 & 0.7059 \\
\hline CD4 (cells $\left./ \mathrm{mm}^{3}\right)$ & $264.50 \pm 84.00$ & $322.20 \pm 102.80$ & $233.10 \pm 103.2$ & $429.50 \pm 124.1$ & 0.211 & 0.1693 \\
\hline CD3 (cells $/ \mathrm{mm}^{3}$ ) & $1029.00 \pm 425.20$ & $1154.00 \pm 401.00$ & $1021.00 \pm 426.1$ & $1478.00 \pm 452.10$ & 0.1263 & 0.0519 \\
\hline $\mathrm{CD} 4 / \mathrm{CD} 3$ & $0.23 \pm 0.07$ & $0.25 \pm 0.07$ & $0.19 \pm 0.04$ & $0.29 \pm 0.09$ & 0.2379 & 0.4632 \\
\hline $\mathrm{WBC}\left(10^{3} / \mu \mathrm{L}\right)$ & $5.06 \pm 2.00$ & $5.31 \pm 0.29$ & $5.57 \pm 1.39$ & $4.72 \pm 1.20$ & 0.6036 & 0.7046 \\
\hline \multicolumn{7}{|l|}{ Haematological profile } \\
\hline $\mathrm{Hb}(\mathrm{g} / \mathrm{dL})$ & $9.32 \pm 1.17$ & $10.73 \pm 2.19$ & $8.89 \pm 1.35$ & $11.75 \pm 1.11$ & $<0.0001$ & 0.0008 \\
\hline $\operatorname{RBC}\left(10^{6} / \mu \mathrm{L}\right)$ & $3.43 \pm 0.52$ & $3.86 \pm 0.72$ & $3.45 \pm 0.56$ & $3.79 \pm 0.58$ & 0.0016 & 0.2581 \\
\hline HCT (\%) & $28.89 \pm 3.56$ & $32.66 \pm 6.13$ & $27.60 \pm 3.80$ & $34.29 \pm 3.46$ & $<0.0001$ & 0.0155 \\
\hline MCV (fL) & $85.41 \pm 12.13$ & $85.70 \pm 12.80$ & $81.35 \pm 12.36$ & $92.12 \pm 14.50$ & 0.0954 & 0.1948 \\
\hline $\mathrm{MCH}(\mathrm{pg})$ & $28.39 \pm 5.30$ & $29.18 \pm 5.39$ & $27.18 \pm 5.54$ & $31.72 \pm 4.96$ & 0.0785 & 0.1214 \\
\hline $\mathrm{MCHC}(\mathrm{g} / \mathrm{dL})$ & $33.24 \pm 3.21$ & $34.05 \pm 3.04$ & $33.36 \pm 3.37$ & $34.51 \pm 1.15$ & 0.3722 & 0.2879 \\
\hline RDW-CV & $16.61 \pm 2.16$ & $15.76 \pm 2.18$ & $16.76 \pm 2.08$ & $14.98 \pm 1.47$ & 0.0161 & 0.0579 \\
\hline RDW-SD & $52.59 \pm 8.49$ & $50.98 \pm 6.86$ & $50.66 \pm 7.52$ & $51.02 \pm 7.05$ & 0.6319 & 0.4892 \\
\hline $\operatorname{PLT}\left(10^{3} / \mu \mathrm{L}\right)$ & $251.50 \pm 91.12$ & $252.80 \pm 95.10$ & $270.60 \pm 88.36$ & $220.30 \pm 97.97$ & 0.461 & 0.3957 \\
\hline \multicolumn{7}{|l|}{ Biochemical profile } \\
\hline Iron $(\mu \mathrm{mol} / \mathrm{L})$ & $8.93 \pm 2.98$ & $4.60 \pm 1.82$ & $4.01 \pm 2.07$ & $49.47 \pm 19.94$ & $<0.0001$ & $<0.0001$ \\
\hline Ferritin $(\mu \mathrm{g} / \mathrm{L})$ & $229.00 \pm 55.13$ & $223.80 \pm 53.00$ & $248.90 \pm 62.32$ & $193.10 \pm 16.69$ & 0.0215 & 0.1106 \\
\hline Transferrin $(\mathrm{mg} / \mathrm{dL})$ & $290.80 \pm 70.02$ & $284.20 \pm 67.31$ & $316.10 \pm 79.14$ & $245.20 \pm 21.20$ & 0.0215 & 0.1106 \\
\hline TIBC $(\mu \mathrm{g} / \mathrm{dL})$ & $18.87 \pm 7.49$ & $9.60 \pm 2.96$ & $7.71 \pm 2.24$ & $112.80 \pm 47.94$ & $<0.0001$ & $<0.0001$ \\
\hline
\end{tabular}

$P$ values $<0.05$ were considered significant. $P$ values represent one-way ANOVA of all variables. $\mathrm{WBC}=$ White Blood Cells, $\mathrm{Hb}=$ haemoglobin, RBC $=\mathrm{Red}$ Blood Cells, HCT = haematocrit, $\mathrm{MCV}=$ Mean Cell Volume, $\mathrm{MCH}=$ Mean Cell Haemoglobin, $\mathrm{MCHC}=$ Mean Cell Haemoglobin Concentration, RDW-CV = Red Cell Distribution Width-Cumulative Variance, RDW-SD = Red Cell Distribution Width-Standard Deviation, PLT = platelets, TIBC = Total Iron Binding Capacity, TSAT $=$ transferrin saturation, and HAART $=$ Highly Active Antiretroviral Therapy.

TABLE 7: Anaemia and associated risks, amongst the study population.

\begin{tabular}{|c|c|c|c|c|}
\hline Parameter & On-HAART $(n=219)$ & HAART-naive $(n=100)$ & OR (95\% CI) & $P$ value \\
\hline \multicolumn{5}{|l|}{ WHO/ACTG } \\
\hline Grade $1 n(\%)$ & $20(9.1)$ & $21(21.0)$ & $2.6(1.3595$ to 5.1458$)$ & 0.0042 \\
\hline Grade $2 n(\%)$ & $13(5.9)$ & $6(6.0)$ & $1.0(0.373$ to 2.743$)$ & 0.9821 \\
\hline Grade $3 n(\%)$ & $7(3.2)$ & $9(9.0)$ & 3.0 (1.082 to 8.289$)$ & 0.0346 \\
\hline
\end{tabular}

WHO/ACTG = World Health Organization/AIDS Clinical Trial Group; OR = Odds Ratio, and HAART = Highly Active Antiretroviral Therapy. Reference = On-HAART.

In the present study, prevalence of anaemia in the total population was $23.8 \%$. Our prevalence is similar to that observed in advanced countries [22, 33] but significantly lower than that observed by other researchers in neighbouring African countries [11, 34, 35]. In a recent study conducted in Tanzania, a prevalence of $77.4 \%$ was recorded and this was attributed to advanced immunodeficiency and the high prevalence of anaemia in the African subregion [11]. We however believe the observed prevalence of anaemia may be due to the high proportion of On-HAART individuals. In support, a study conducted in Tanzania showed that anaemia was far more frequent in $\mathrm{HIV}$-infected adults than in their HIV-negative counterparts [36].

We found that anaemia was consistent with increasing disease progression of $\mathrm{HIV}$ as per recommendations of the Center for Disease Control (CDC) [27-29]. Similarly, anaemia at the time of HAART initiation has been associated with HIV disease progression and mortality [6, 8-12]. This association has been linked to an increasing viral burden as HIV disease progresses and may cause anaemia through increased cytokine-mediated myelosuppression. HAART on the other hand has been shown to significantly treat anaemia of HIV infection [21-23], thus reducing the incidence of anaemia in HIV. This is in line with our observation that the frequency of anaemia was higher amongst participants not on HAART. HAART in itself may have a protective effect on the development of anaemia, through reduced disease progression. Further evidence from a study conducted in Tanzania showed that haemoglobin level increased significantly at $2.5 \mathrm{~g} / \mathrm{dL}$ over the first 12 months in patients who received HAART [11]. 
With the complexity of HIV disease, however, anaemia may be a surrogate marker of some other underlying condition, which may not be captured through categorization with CD4 lymphocyte counts and clinical AIDS diagnosis alone [33]. Furthermore, anaemia can be a feature of certain opportunistic diseases [37] and other etiologic factors including micronutrient deficiencies and blood loss from intestinal opportunistic disease [14, 37], a common feature in the developing world. This is consistent with our observation that haemoglobin concentration, serum iron, ferritin, and TSAT decreased from grade 1 to grade 3 anaemia. Iron supplementation is however feared, as adverse effects of iron excess in HIV-infected individuals have been reported in industrialized countries [38]. This is similar to our observation that the mean levels of iron stores were within normal limits.

It is thought that the predominant cause of anaemia in the context of HIV is anaemia of inflammation (AI), also known as anaemia of chronic disease [1]. The observed WBC count was higher amongst the HAART-naive individuals, who had a higher prevalence and frequency of anaemia. It is noteworthy that although inflammation may play a role in the higher prevalence of anaemia amongst the HAART-naive individuals, WBC values were within normal reference limits and we measured CRP to rule out all participants who had active inflammation. Furthermore, immunological markers decreased significantly with increasing severity of disease (Table 5). In this instance, anaemia of chronic disease may be a more appropriate term for our study participants rather than anaemia of chronic inflammation.

Serum iron, ferritin, and TSAT were significantly lower in HIV patients with anaemia than in those without anaemia. Iron supplementation has been shown to reduce anaemia in $\mathrm{HIV}$ patients, without adverse effects on $\mathrm{HCV}$ coinfection or plasma HIV-RNA [37]. Notwithstanding, it has been suggested that care must be taken in iron supplementation, to prevent adverse effects of iron excess in HIV-infected individuals [38]. The present study is in agreement with this suggestion as the mean levels of these markers of iron stores for all participants (with HIV) were within normal limits. Thus, in this case, iron deficiency may not be a key player in anaemia in HIV.

In this study, we used haemoglobin as measure of anaemia. Haemoglobin measurement plays an important role in the basic management of HIV disease in West Africa [39] and could be measured easily where resources for more sophisticated laboratory markers are not available. For better monitoring and intervention, regular measurements could be of great help.

\section{Conclusion}

In this population, HIV-associated anaemia is common. Anaemia was more prevalent in severe disease forms and the frequency of anaemia was higher in participants yet to initiate HAART. High serum ferritin, transferrin, and TIBC in severe disease forms suggest low iron distribution in HIV infection and that HIV infection does not adversely influence tissue iron availability. The present study confirms that HIV itself is the most important cause of anaemia and that iron deficiency is of less importance. The treatment of HIV should be a priority in management strategies compared to iron supplementation.

\section{Competing Interests}

The authors declare that they have no competing interests.

\section{Acknowledgments}

Gratitude goes to the staff at the Sexually Transmitted Infections Clinic of the Tamale Teaching Hospital. The authors appreciate all patients who volunteered to be part of this study, making it a huge success.

\section{References}

[1] K. Tolentino and J. F. Friedman, "An update on anemia in less developed countries," The American Journal of Tropical Medicine and Hygiene, vol. 77, no. 1, pp. 44-51, 2007.

[2] R. Yip and U. Ramakrishnan, "Experiences and challenges in developing countries," Journal of Nutrition, vol. 132, supplement 4, pp. 827S-830S, 2002.

[3] WHO, Worldwide Prevalence of Anaemia 1993-2005, World Health Organization, Geneva, Switzerland, 2008.

[4] C. E. Bekolo, M. B. Nguena, L. Ewane, P. S. Bekoule, and B. Kollo, "The lipid profile of HIV-infected patients receiving antiretroviral therapy in a rural Cameroonian population," BMC Public Health, vol. 14, no. 1, article 236, 2014.

[5] UNAIDS, Global Report: UNAIDS Report on the Global AIDS Epidemic 2013, 2013.

[6] P. S. Belperio and D. C. Rhew, "Prevalence and outcomes of anemia in individuals with human immunodeficiency virus: a systematic review of the literature," American Journal of Medicine, vol. 116, supplement 7, pp. 27-43, 2004.

[7] R. J. Harris, J. A. C. Sterne, S. Abgrall et al., "Prognostic importance of anaemia in HIV type-1-infected patients starting antiretroviral therapy: collaborative analysis of prospective cohort studies," Antiviral Therapy, vol. 13, no. 8, pp. 959-967, 2008.

[8] M. May, A. Boulle, S. Phiri et al., "Prognosis of patients with HIV-1 infection starting antiretroviral therapy in sub-Saharan Africa: a collaborative analysis of scale-up programmes," The Lancet, vol. 376, no. 9739, pp. 449-457, 2010.

[9] J. S. A. Stringer, I. Zulu, J. Levy et al., "Rapid scale-up of antiretroviral therapy at primary care sites in Zambia: feasibility and early outcomes," The Journal of the American Medical Association, vol. 296, no. 7, pp. 782-793, 2006.

[10] J.-F. Etard, I. Ndiaye, M. Thierry-Mieg et al., "Mortality and causes of death in adults receiving highly active antiretroviral therapy in Senegal: a 7-year cohort study," AIDS, vol. 20, no. 8, pp. 1181-1189, 2006.

[11] A. Johannessen, E. Naman, B. J. Ngowi et al., "Predictors of mortality in $\mathrm{HIV}$-infected patients starting antiretroviral therapy in a rural hospital in Tanzania," BMC Infectious Diseases, vol. 8, article 52, 2008.

[12] M. E. O’Brien, R. Kupka, G. I. Msamanga, E. Saathoff, D. J. Hunter, and W. W. Fawzi, "Anemia is an independent predictor of mortality and immunologic progression of disease among women with HIV in Tanzania," Journal of Acquired Immune Deficiency Syndromes, vol. 40, no. 2, pp. 219-225, 2005. 
[13] C. Obirikorang and F. A. Yeboah, "Blood haemoglobin measurement as a predictive indicator for the progression of HIV/AIDS in resource-limited setting," Journal of Biomedical Science, vol. 16, no. 1, article 102, 2009.

[14] J. G. Shaw and J. F. Friedman, "Iron deficiency anemia: focus on infectious diseases in lesser developed countries," Anemia, vol. 2011, Article ID 260380, 10 pages, 2011.

[15] J. M. McDermid, A. Jaye, M. F. Schim Van Der Loeff et al., "Elevated iron status strongly predicts mortality in West African adults with HIV infection," Journal of Acquired Immune Deficiency Syndromes, vol. 46, no. 4, pp. 498-507, 2007.

[16] A. S. W. Mburu, D. I. Thurnham, D. L. Mwaniki, E. M. Muniu, F. Alumasa, and A. De Wagt, "The influence and benefits of controlling for inflammation on plasma ferritin and hemoglobin responses following a multi-micronutrient supplement in apparently healthy, HIV+ Kenyan adults," Journal of Nutrition, vol. 138, no. 3, pp. 613-619, 2008.

[17] M. A. Salomé and H. Z. W. Grotto, "Human immunodeficiency virus-related anemia of chronic disease: relationship to hematologic, immune, and iron metabolism parameters, and lack of association with serum interferon- $\gamma$ levels," AIDS Patient Care and STDs, vol. 16, no. 8, pp. 361-365, 2002.

[18] J. Boom, E. Kösters, C. Duncombe et al., "Ferritin levels during structured treatment interruption of highly active antiretroviral therapy," HIV Medicine, vol. 8, no. 6, pp. 388-395, 2007.

[19] A. Costantini, S. Giuliodoro, L. Butini, G. Silvestri, P. Leoni, and M. Montroni, "Abnormalities of erythropoiesis during HIV-1 disease: a longitudinal analysis," Journal of Acquired Immune Deficiency Syndromes, vol. 52, no. 1, pp. 70-74, 2009.

[20] P. A. Volberding, A. M. Levine, D. Dieterich, D. Mildvan, R. Mitsuyasu, and M. Saag, "Anemia in HIV infection: clinical impact and evidence-based management strategies," Clinical Infectious Diseases, vol. 38, no. 10, pp. 1454-1463, 2004.

[21] A. Mocroft, O. Kirk, S. E. Barton et al., "Anaemia is an independent predictive marker for clinical prognosis in HIV-infected patients from across Europe," AIDS, vol. 13, no. 8, pp. 943-950, 1999.

[22] R. D. Moore and D. Forney, "Anemia in HIV-infected patients receiving highly active antiretroviral therapy," Journal of Acquired Immune Deficiency Syndromes, vol. 29, no. 1, pp. 5457, 2002.

[23] K. Berhane, R. Karim, M. H. Cohen et al., "Impact of highly active antiretroviral therapy on anemia and relationship between anemia and survival in a large cohort of HIV-infected women: women's interagency HIV study," Journal of Acquired Immune Deficiency Syndromes, vol. 37, no. 2, pp. 1245-1252, 2004.

[24] C. Obirikorang, P. K. Selleh, J. K. Abledu, and C. O. Fofie, "Predictors of adherence to antiretroviral therapy among HIV/AIDS patients in the upper west region of Ghana," ISRN AIDS, vol. 2013, Article ID 873939, 7 pages, 2013.

[25] T. Leenstra, S. K. Kariuki, J. D. Kurtis, A. J. Oloo, P. A. Kager, and F. O. ter Kuile, "Prevalence and severity of anemia and iron deficiency: cross-sectional studies in adolescent schoolgirls in western Kenya," European Journal of Clinical Nutrition, vol. 58, no. 4, pp. 681-691, 2004.

[26] A. M. Konijn, "Iron metabolism in inflammation," Baillière's Clinical Haematology, vol. 7, no. 4, pp. 829-849, 1994.

[27] CDC, "1992 revised classification system for HIV infection and expanded surveillance case definition for AIDS among adolescents and adults," Morbidity and Mortality Weekly Report, vol. 41, no. 17, pp. 961-962, 1993.
[28] CDC, "Revised guidelines for performing CD4+ T-cell determinations in persons infected with human immunodeficiency virus (HIV)," Morbidity and Mortality Weekly Report, vol. 46, no. 2, pp. 1-29, 1997.

[29] F. F. Mandy, J. K. A. Nicholson, and J. S. McDougal, “Guidelines for performing single-platform absolute $\mathrm{CD} 4^{+} \mathrm{T}$-cell determinations with CD45 gating for persons infected with human immunodeficiency virus," Morbidity and Mortality Weekly Report, vol. 52, no. 2, pp. 1-13, 2003.

[30] G. Moyle, "Anaemia in persons with HIV infection: prognostic marker and contributor to morbidity," AIDS Reviews, vol. 4, no. 1, pp. 13-20, 2002.

[31] R. Wisaksana, R. Sumantri, A. R. Indrati et al., "Anemia and iron homeostasis in a cohort of HIV-infected patients in Indonesia," BMC Infectious Diseases, vol. 11, article 213, 2011.

[32] WHO, Iron Deficiency Anaemia: Assessment, Prevention and Control, 2001, http://apps.who.int/iris/bitstream/10665/66914/1/ WHO_NHD_01.3.pdf.

[33] P. S. Sullivan, D. L. Hanson, S. Y. Chu, J. L. Jones, and J. W. Ward, "Epidemiology of anemia in human immunodeficiency virus (HIV)-infected persons: results from the multistate adult and adolescent spectrum of HIV disease surveillance project," Blood, vol. 91, no. 1, pp. 301-308, 1998.

[34] O. Erhabor, O. A. Ejele, C. A. Nwauche, and F. I. Buseri, "Some haematological parameters in Human Immunodeficiency Virus (HIV) infected Africans: the Nigerian perspective," Nigerian Journal of Medicine, vol. 14, no. 1, pp. 33-38, 2005.

[35] R. Omoregie, E. U. Omokaro, O. Palmer et al., "Prevalence of anaemia among HIV-infected patients in Benin City, Nigeria," Tanzania Journal of Health Research, vol. 11, no. 1, pp. 1-4, 2009.

[36] B. J. Ngowi, S. G. Mfinanga, J. N. Bruun, and O. Morkve, "Immunohaematological reference values in human immunodeficiency virus-negative adolescent and adults in rural northern Tanzania," BMC Infectious Diseases, vol. 9, no. 1, pp. 1-7, 2009.

[37] R. D. Semba and G. E. Gray, "Pathogenesis of anemia during human immunodeficiency virus infection," Journal of Investigative Medicine, vol. 49, no. 3, pp. 225-239, 2001.

[38] A. Savarino, G. P. Pescarmona, and J. R. Boelaert, "Iron metabolism and HIV infection: reciprocal interactions with potentially harmful consequences?" Cell Biochemistry and Function, vol. 17, no. 4, pp. 279-287, 1999.

[39] E. Ledru, S. Diagbouga, N. Meda et al., "A proposal for basic management of HIV disease in West Africa: use of clinical staging and haemogram data," International Journal of STD and AIDS, vol. 9, no. 8, pp. 463-470, 1998. 


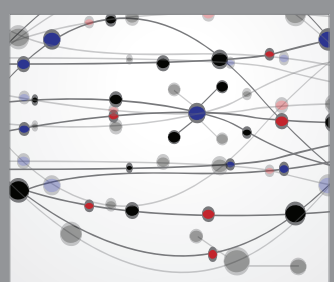

The Scientific World Journal
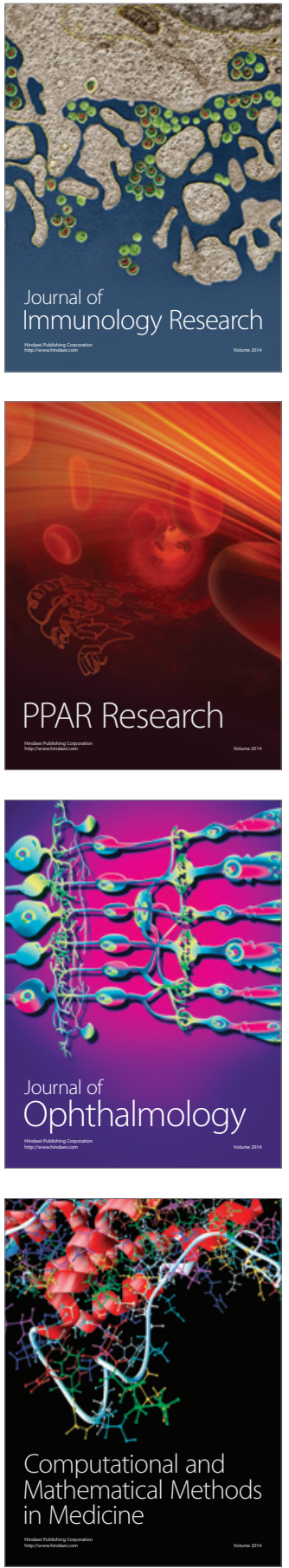

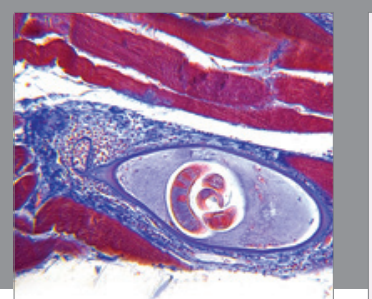

Gastroenterology Research and Practice

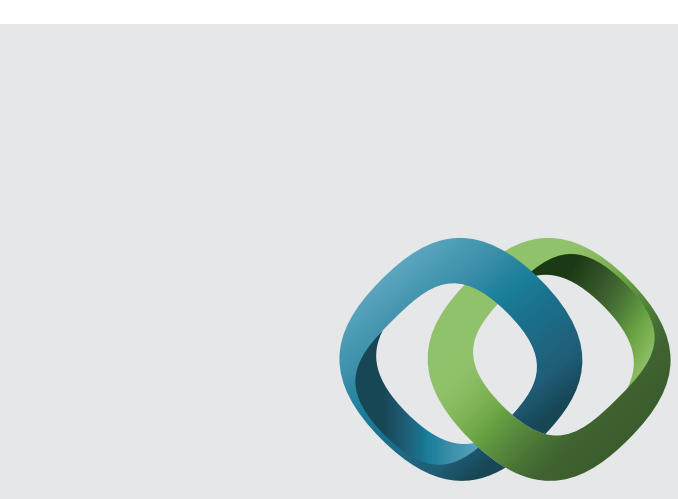

\section{Hindawi}

Submit your manuscripts at

http://www.hindawi.com
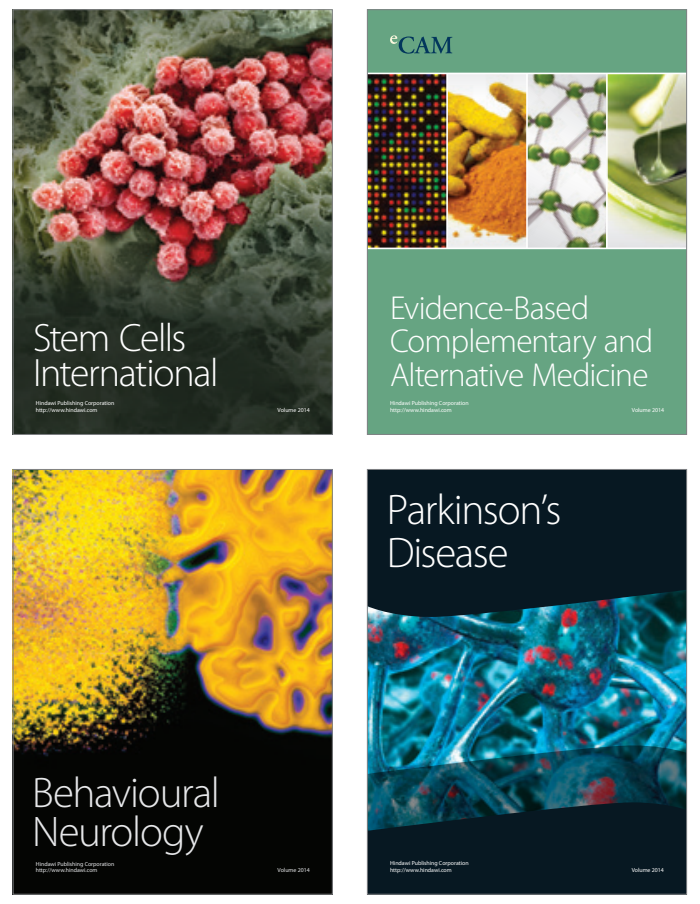
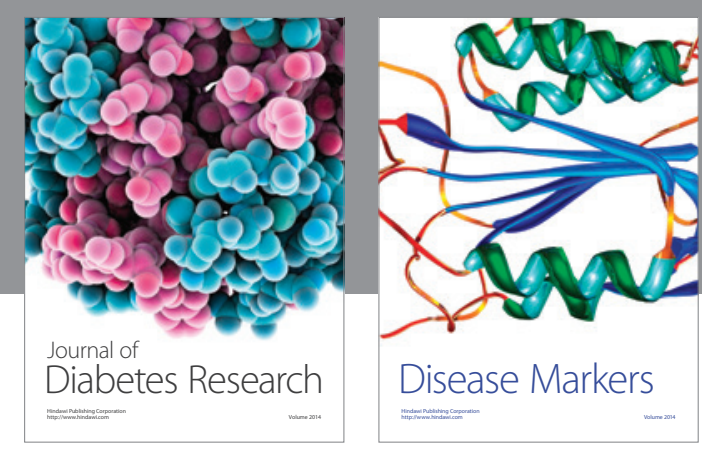

Disease Markers
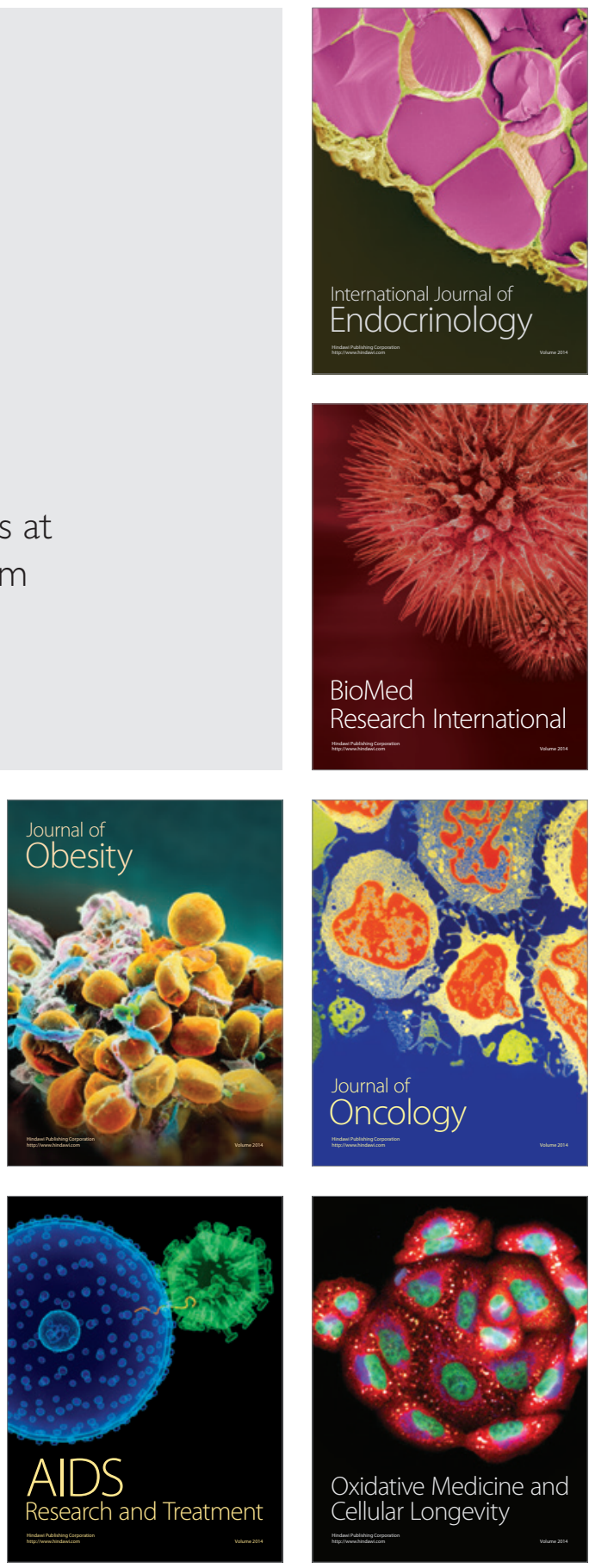